\title{
New pulsar discoveries from re-analysis of Parkes Multibeam survey candidates
}

\author{
Michael Keith*, Andrew Lyne, Michael Kramer \\ $J B C A /$ University of Manchester \\ E-mail: mkeith@pulsarastronomy . net
}

\begin{abstract}
The Parkes Multibeam Pulsar Survey is the most successful pulsar survey to date, discovering more than 700 previously unknown pulsars. Re-analysis of the candidates produced by the survey using new software tools, including a heuristic scoring system, has discovered a further 30 pulsars. These include a young pulsar, spatially co-incident with a supernova remnant, and an eccentric binary.
\end{abstract}

From Planets to Dark Energy: the Modern Radio Universe

October 1-5 2007

The University of Manchester, UK

\footnotetext{
* Speaker.
} 


\section{Introduction: The Parkes Multibeam Pulsar Survey}

The Parkes Multibeam Pulsar Survey (PMPS) is the most successful pulsar survey to date, discovering more than 700 of the 2000 known pulsars [1-4]. The survey was carried out at the Parkes Telescope in Australia, using a new 13-beam receiver operating at $1400 \mathrm{MHz}$. A 10 by 150 degree strip of the galactic plane was covered generating over 4 TB of data. The data was initially processed at several locations, then in 2002 the data was collected at Jodrell Bank and re-processed using more advanced algorithms [5].

\section{New Techniques}

JReaper is a new graphical pulsar candidate selection tool. Based on a previous system [5], this allows for the millions of candidates produced in pulsar surveys to be viewed in a simple, customisable plot. For example, candidates can be shown on a plane of pulse period against signalto-noise ratio. By selecting candidates with the cursor, the full detailed plots are presented to the user. JReaper includes several advanced features, of particular note is the pulsar candidate scoring system. This system scores the various aspects of the candidate signal, with pulsar-like signals scoring highly. As an example, the variation of signal-to-noise ratio with trial dispersion measure is compared to a theoretically computed curve, and the level of deviation determines the score. Similarly the variation of signal-to-noise with trial folding period can be used.

These scores are then combined with a weighted average and can be used on one of the axes of the JReaper plot. This provides an alternative way to view the data that can separate pulsars from interference signals with similar period. Other JReaper enhancements include a system for accurately marking or hiding known pulsars and their harmonics, the ability to colour data points depending on a given parameter (e.g. DM) and the ability to view multiple candidates sequentially.

\section{New Pulsars}

Application of JReaper to the candidates from the 2002 processing of the PMPS has led to the discovery of 30 previously unknown pulsars. These pulsars have been discovered without any new observations nor substantial computing resource. Full details of the discovered pulsars will be published when a coherent timing solution for each has been obtained.

\subsection{J1849-0020: A young pulsar with a possible SNR association}

The discovered pulsar J1849-0020 has a period of $166 \mathrm{~ms}$ and a characteristic age of 67 kyr. Given this age, the remnant of the supernova explosion that formed the pulsar could still be detectable. The Green supernova remnant catalogue [7] lists a spatially coincident source, G32.45+0.1. This source has been observed in the X-ray band with XMM-Newton [8] and is detected in the NVSS L-band VLA survey [9]. Distance measurements to the pulsar (using the NE2001 electron density model [10]) and to the remnant (using Hydrogen absorption [8]) agree within the errors to be $\sim 11 \pm 5 \mathrm{kpc}$. Given the estimated age of the pulsar and a separation of $\sim 3^{\prime}$, the pulsar transverse velocity must be $200 \mathrm{~km} / \mathrm{s}$, well within the observed pulsar distribution. Long term timing of the source should allow for measurement of a proper motion, which if the association is true, should be away from the remnant centre. 


\subsection{J1753-2243: A pulsar in an eccentric binary system}

Timing observations of J1753-2243 revealed that the pulsar was in an eccentric binary system with an orbital period of 13.6 days and eccentricity of 0.3 . The pulsar has a rotation period of $95 \mathrm{~ms}$, however its spin down rate is less than $2 \times 10^{-18}$ giving a characteristic age of greater than $500 \mathrm{Myr}$. These parameters are indicative of a pulsar that has undergone a short phase of accretion induced spin-up, implying that the companion was a massive star that underwent a supernova explosion. Although the minimum companion mass is small, it seems likely that the companion is a neutron star. Identification of the companion type may be aided by measurement of relativistic binary parameters such as advance of periastron, as these can be used to constrain the masses. Simulated observations suggest that this could be measured in $\sim 7$ years. Searches for a companion pulsar have not shown any periodic signal down to a limit of $\sim 0.1 \mathrm{mJy}$.

\section{Conclusion}

Modern pulsar surveys produce far more candidates than can be individually inspected. Improved selection methods can improve detection of pulsars by increasing the likelihood that a good candidate pulsar is inspected. Application of improved techniques to existing PMPS results has yielded 30 previously unknown pulsars. These include an eccentric binary, which may be the 10th known double neutron star system, and a pulsar spatially coincident with a known supernova remnant.

\section{References}

[1] R. N. Manchester, et al., The Parkes multi-beam pulsar survey - I. Observing and data analysis systems, discovery and timing of 100 pulsars, MNRAS 328 (2001).

[2] D. J. Morris, et al., The Parkes Multibeam Pulsar Survey - II. Discovery and timing of 120 pulsars, MNRAS 335 (2002).

[3] M. Kramer, et al., The Parkes Multibeam Pulsar Survey - III. Young pulsars and the discovery and timing of 200 pulsars, MNRAS 342 (2003).

[4] G. Hobbs, et al., The Parkes multibeam pulsar survey - IV. Discovery of 180 pulsars and parameters for 281 previously known pulsars, MNRAS 352 (2004).

[5] A. J. Faulkner, et al., The Parkes Multibeam Pulsar Survey - V. Finding binary and millisecond pulsars, MNRAS 355 (2004).

[6] D. R. Lorimer, et al., The Parkes Multibeam Pulsar Survey - VI. Discovery and timing of 142 pulsars and a Galactic population analysis, MNRAS 372 (2006)

[7] D. A. Green, A Catalogue of Galactic Supernova Remnants (2006 April Version), http://www.mrao.cam.ac.uk/surveys/snrs/ (2006).

[8] H. Yamaguchi, et al., XMM-Newton Observations of G32.45+0.1 and G38.55+0.0: Diffuse Hard X-Ray Sources Found by the ASCA Galactic Plane Survey, PASJ 56 (2004).

[9] J. J. Condon, et al., The NRAO VLA Sky Survey, AJ 115 (1998).

[10] J. M. Cordes and T. J. W. Lazio, NE2001.I. A New Model for the Galactic Distribution of Free Electrons and its Fluctuations, astro-ph/0207156v3. 